The Radiochemical Analysis of Gaseous Samples (RAGS) Apparatus for Nuclear Diagnostics at the National Ignition Facility

D. A. Shaughnessy, C. A. Velsko, D. R. Jedlovec, C. B. Yeamans, K. J. Moody, E. Tereshatov, W. Stoeffl, A. Riddle

May 14, 2012

19th Topical Conference High-Temperature Plasma Diagnostics

Monterey, CA, United States

May 6, 2012 through May 10, 2012 
This document was prepared as an account of work sponsored by an agency of the United States government. Neither the United States government nor Lawrence Livermore National Security, LLC, nor any of their employees makes any warranty, expressed or implied, or assumes any legal liability or responsibility for the accuracy, completeness, or usefulness of any information, apparatus, product, or process disclosed, or represents that its use would not infringe privately owned rights. Reference herein to any specific commercial product, process, or service by trade name, trademark, manufacturer, or otherwise does not necessarily constitute or imply its endorsement, recommendation, or favoring by the United States government or Lawrence Livermore National Security, LLC. The views and opinions of authors expressed herein do not necessarily state or reflect those of the United States government or Lawrence Livermore National Security, LLC, and shall not be used for advertising or product endorsement purposes. 


\title{
The Radiochemical Analysis of Gaseous Samples (RAGS) Apparatus for Nuclear Diagnostics at the National Ignition Facilitya)
}

\author{
D. A. Shaughnessy ${ }^{\text {b) }}$, C. A. Velsko, D. R. Jedlovec, C. B. Yeamans, K. J. Moody, \\ E. Tereshatov, W. Stoeffl, and A. Riddle \\ Lawrence Livermore National Laboratory, PO Box 808, L-236, Livermore, CA, 94551, USA
}

(Presented May 8, 2012; received XXXXX; accepted XXXXX; published online XXXXX)

\begin{abstract}
The RAGS (Radiochemical Analysis of Gaseous Samples) diagnostic apparatus was recently installed at the National Ignition Facility. Following a NIF shot, RAGS is used to pump the gas load from the NIF chamber for purification and isolation of the noble gases. After collection, the activated gaseous species are counted via gamma spectroscopy for measurement of the capsule areal density and fuel-ablator mix. Collection efficiency was determined by injecting a known amount of ${ }^{135} \mathrm{Xe}$ into the NIF chamber, which was then collected with RAGS. Commissioning was performed with an exploding pusher capsule filled with isotopically enriched ${ }^{124} \mathrm{Xe}$ and ${ }^{126} \mathrm{Xe}$ added to the DT gas fill. Activated xenon species were recovered postshot and counted via gamma spectroscopy. Results from the collection and commissioning tests are presented. The performance of RAGS allows us to establish a noble gas collection method for measurement of noble gas species produced via neutron and charged particle reactions in a NIF capsule.
\end{abstract}

\section{INTRODUCTION}

Radiochemical diagnostic methods are currently being implemented for the National Ignition Facility (NIF). The need to diagnose NIF target performance can be met through the addition of radiochemical detectors to the capsule, which have the unique capability to be placed in the target fill gas in the case of an exploding pusher shell or in the ablator shell in a cryogenic layered implosion target. Radiochemistry samples in the gasphase offer a direct method of collection if the target chamber is pumped out following a NIF shot and the gas is transported downstream for further purification and analysis. The Radiochemical Analysis of Gaseous Samples (RAGS) apparatus was designed for the purpose of collecting and analyzing gases produced via the ignition process.

The RAGS apparatus is a two-part system consisting of a pre-filter, which removes water vapor, particulates, and reactive gases, and the second part, which consists of a cryogenic noble gas collection system. RAGS was built and tested by Sandia National Laboratory (Albuquerque) in collaboration with NIF. It is planned for use during the National Ignition Campaign to determine the areal density ( $\rho r)$ of the NIF ignition capsule, and there is also the possibility of using it to measure mix between the ablator and the fuel. Small numbers of dopant atoms (roughly $10^{15}$ ) will be added to the inner-most layers of the capsule ablator shell. This number of dopant atoms has been shown through simulation to not affect the performance of a typical ignition capsule. $^{1}$ These elements will undergo nuclear activation from neutrons, deuterons, or alpha particles produced via the fusion process. Following a shot, the activated gases will be pumped out of the chamber and transported to the RAGS apparatus. The subsequent isotopic ratios that are measured in the sample can then be correlated to the fraction of downscattered neutrons that originated from the NIF capsule, and consequently the pr. Commissioning has been performed with an exploding pusher shell containing a 50:45 mix of isotopically enriched ${ }^{124} \mathrm{Xe}$ and

\footnotetext{
a) Invited paper published as part of the Proceedings of the 19th Topical Conference on High-Temperature Plasma Diagnostics, Monterey, California, May, 2012.

b) Author to whom correspondence should be addressed: shaughnessy2@1lnl.gov
}

${ }^{126} \mathrm{Xe}$ (with approximately $5 \%{ }^{128} \mathrm{Xe}$ ) added to the DT gas fill. For the first cryogenic ignition capsule experiments, ${ }^{124} \mathrm{Xe}$ will be doped in the first 6 microns of the plastic ablator shell. The ratio of the neutron capture product $\left({ }^{125} \mathrm{Xe}\right)$ to the $(\mathrm{n}, 2 \mathrm{n})$ activation product $\left({ }^{123} \mathrm{Xe}\right)$ will provide capsule designers important diagnostic information related to the areal density of the capsule. In a beryllium capsule, ${ }^{127}$ I could also be co-doped with the ${ }^{124} \mathrm{Xe}$; $(\mathrm{d}, 2 \mathrm{n})$ reactions on the iodine would form ${ }^{127} \mathrm{Xe}$, which would then be an indicator of mix. Following the NIF shot, the gases from the chamber will be pumped to the RAGS apparatus for purification and isolation of the xenon noble gas fraction. The xenon is ultimately collected on a cryogenic cold head and then released into a sample bottle for subsequent gamma spectroscopy and/or mass spectrometry.

Collection efficiency has been determined through the injection of a known amount of ${ }^{135} \mathrm{Xe}$ into the NIF chamber with subsequent collection and measurement of the residual xenon gas fraction. Results from the efficiency tests and the first commissioning shot using a xenon filled exploding pusher shell will be presented. Results from the first RAGS experiments have demonstrated that the performance of RAGS is sufficient to use the instrument for measurement of $\rho$ r and capsule mix.

\section{RAGS EQUIPMENT}

The modular design concept for the RAGS system uses multiple carts to process gas from the NIF target chamber and isolate individual noble gases for transfer to in situ gamma detectors. In operation, the NIF cryopumps are isolated just before shot time and the target chamber turbopumps alone deliver gas to the RAGS sampling line. The first module, a filter cart, removes water vapor, hydrogen and most air gases, leaving a noble gas fraction. The plan calls for a set of serially-connected carts downstream from the filter cart to collect one noble gas in 
each, for in-situ decay counting analysis. As of May 2012, the xenon cart is built, but it does not yet have an in-situ germanium detector. To demonstrate proof of capability, we collect xenon in a removable sample bottle and use an LLNL Nuclear Counting Facility germanium detector for decay counting. On one test using radioactive xenon injected into the target chamber, the RAGS sample bottle contained $63 \%$ of the initial gas amount. FIG. 1 shows the complete RAGS instrument.

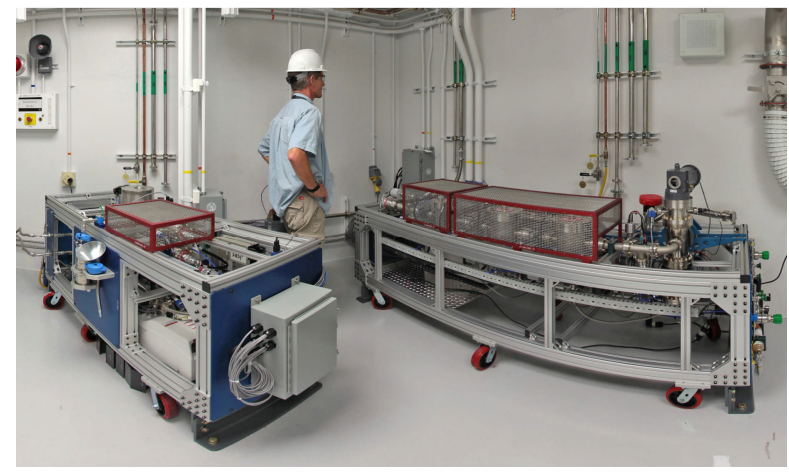

FIG. 1. The RAGS System as deployed at the National Ignition Facility. Gas from the NIF target chamber enters the filter cart on the right. The filter cart removes water, particulates, and other reactive gases so that only the noble gases enter the collection cart on the left, where noble gases are cryogenically fractionated.

\section{A. RAGS Control}

All of the RAGS components are controlled through NIF Operations interfaces. RAGS activities occur in three timeframes: pre-shot, when operators bring the carts to "ready for shot" status following a procedure; in-shot, when the sampling and gas processing functions are carried out automatically in sync with the shot timing; and post-shot, when operators retrieve samples and perform cart recovery functions. RAGS valve states and sensor control parameters are set and viewed in the Industrial Control System (ICS) graphical user interfaces representing each cart. Sensor output signals are logged every 4 seconds and archived daily. RAGS LaBr radiation detectors and mass spectrometers are operated through the Integrated Computer Control System (ICCS), which provides customized operation and data collection programs for analytical devices.

\section{B. RAGS Sample Transfer Line}

A 1.5" OD vacuum line connects to the exhaust ring header of the NIF turbo pumps and runs approximately 170 feet to the RAGS room in the -33'6" level of the NIF building. Each of the three NIF turbos is rated at 2,000 liters per minute for nitrogen. At 35 seconds before shot trigger, the cryopumps' gate valves receive a close command to isolate the cryopumps; subsequently at shot time, only the turbopumps are open to the chamber. At 1 second before shot, the valve to the RAGS sampling line is automatically opened and the valve to the turbopump backing pump is closed to direct gas from the chamber to the filter cart. For most of the commissioning and ride-along tests, we have sampled the chamber for 10 minutes, although we can choose the length of time to sample from 0-15 minutes. Using the chamber air leak rate and RAGS pumping speed, we calculate that by Tzero +10 minutes, $90 \%$ of the sample has been directed to RAGS.

Gas transfer time from the turbo ring header to the RAGS filter cart inlet is 20 seconds. During sampling, the pressure rises in the line due to two factors: first, the prompt gas load produced when scattered laser light and energetic radiation from the target release gas from the chamber surfaces; and, second, air leaks into the chamber with a measured rate of 0.5 torr-liter/second. For the low yield shots RAGS has sampled, the pressure at the inlet of the RAGS filter cart rises from 1 to 20 millitorr. During tests without a target shot, there is no measureable pressure rise. In the event that low pressure in the transfer line caused slow gas flow, a helium puff was installed behind the RAGS sampling valve to provide metered flow in continuous or puff modes to carry the sample down the RAGS sample line. Because the sampling line pressures have been high enough to transport gas efficiently we have not used this helium puff. One test measured $88 \%$ yield of xenon gas from an injection behind the RAGS sampling valve to collection in the sample bottle.

\section{RAGS Filter Cart}

The filter cart was designed to process 0.5 torr-liter/second of gas for approximately 10 minutes of sampling time. Given our experience to date, we believe this unit could perform effectively for 20 to 30 minutes sampling. This cart removes water vapor, reactive air components and hydrogen and transfers the noble gas fraction to the collector cart. A Pfeiffer HiPace $80^{2}$ turbopump and an Adixen ACP40 rough pump provide vacuum for the cart and the RAGS Sample Transfer line. The vacuum envelope consists of standard and custom stainless steel vacuum components with knife-edge sealing surfaces (ConFlat ${ }^{\mathrm{TM}}$ ). The cart has a quadrupole mass spectrometer (SRS RGA200) to track the efficiency of the getter units by monitoring gas composition. Clean, dry, heated air for purge and pump operations and a helium puff are available. We have not used this helium puff because the turbo pumps efficiently move gas within the cart and to the next cart. FIG. 2 shows a model of the filter cart.

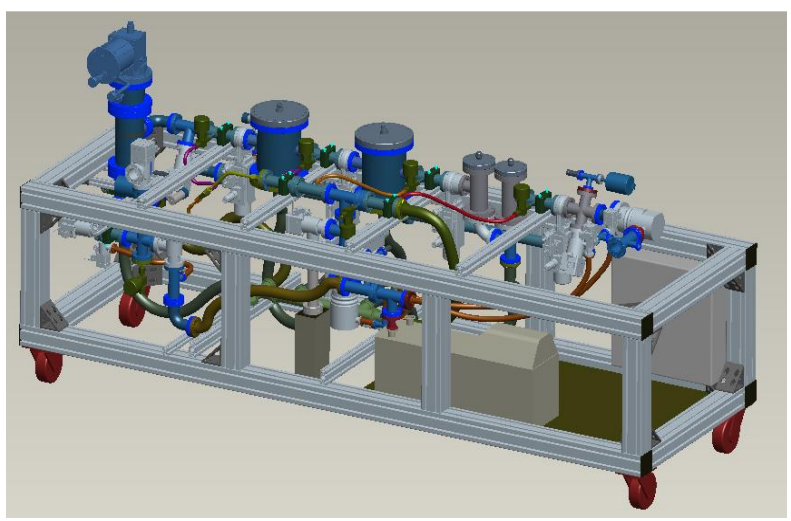

FIG. 2. The RAGS filter cart. Gas enters the water vapor trap on the left-hand side and then flows through the four getters in the center before exiting the filter to travel to the main collection cart. The filter removes water, particulates, and reactive gases so that only the noble gases enter the collection cart. 
The first step in the gas processing is removal of water vapor. The water trap is a custom fabricated assembly consisting of a large-volume, large-surface-area cylinder made from stacked copper foam disks and a solid copper core, mounted on a singlestage Oxford/Austin Scientific M1050 cold head. Custom seals at both ends direct the sample gas through the foam. The unit runs at $155 \mathrm{~K} \pm 0.5 \mathrm{~K}$ in a cool-only mode because the large mass creates substantial thermal inertia that is difficult to warm uniformly with two heaters. During sample processing, a temperature gradient of $+20 \mathrm{~K}$ develops between the inlet and the outlet and the water vapor signal monitored by the filter cart RGA increases $10 \%$

The next step is removal of major air components. The filter cart provides four getters mounted in series. The first two are SAES GP 500 MK5 units maintained at $450^{\circ} \mathrm{C}$ from 1 hour prior to, and until 30 minutes after, the shot. These hot getters remove nitrogen, oxygen and residual water from the sample. Although $450^{\circ} \mathrm{C}$ is above the recommended operating temperature, we have found that this temperature reduces the nitrogen and oxygen a factor of 1000 relative to air abundances. The next two getters are activated SAES GP100 MK5 units used at room temperature for hydrogen absorption. These getters are sized to absorb the residual hydrogen isotopes released from a NIF target and the hydrogen gas generated at shot time (possibly from photochemistry of gases released from surfaces).

The filter cart RGA can sample the gas at the hot getters or at either of the cold getters. We usually monitor composition at the outlet of the last getter. The gas leaving this cart is $>90 \%$ argon, a few percent each nitrogen and water vapor and a trace of hydrogen. The sample gas passes through a 15 foot length of 1.5 " diameter vacuum line to reach the xenon cart.

\section{RAGS Xenon Cart and Abort Tank}

The xenon cart is designed to trap xenon on a cold surface, pump away other gases and transfer the xenon for analysis. A vacuum system with a Pfeiffer HiPace 80 turbo pump and an Adixen ACP15 rough pump generates high vacuum. The vacuum envelope consists of standard and custom stainless steel vacuum components with knife-edge sealing surfaces (ConFlat ${ }^{\mathrm{TM}}$ ). During shot sampling, the turbo exhaust is directed to the closedend 11.4 liter abort tank to isolate and contain any radioactivity until the NIF Tritium Processor System (TPS) can accept effluents. For shot preparation, recovery and maintenance, the turbo exhaust passes through the rough pump to the TPS.

Near the end of the automated sample processing sequence, the valves for two room temperature getters and the RGA are opened. Then the valves for the turbopump and abort tank are closed, leaving the xenon cart isolated and the sample retained on the cryotrap. Once operators can enter the room, they manually control the xenon cart functions, performing the xenon release, transfer to the sample bottle and sample bottle removal. After a germanium detector is installed on the cart, sample transfer and decay counting can be added to the automatic sequence.

All of the gas passes through the xenon cart cryotrap to freeze out xenon. This component is a custom fabricated, flowthrough, variable temperature cryogenic trap composed of a 1.5" long section of copper foam made of stacked discs brazed into the vacuum sleeve; a copper block brazed onto the outside of the sleeve and having attachment points for 4-25 Watt cartridge heaters and 2 RTDs. Two flange and flex sections are welded at 90 degrees to the sleeve to form a U-shape. This piece bolts into a custom fabricated vacuum flange that mates with an Oxford/Austin Scientific Model 350, 2 stage cold head and a custom vacuum housing. We keep the housing at vacuum to provide thermal insulation for the cold head. The first stage of the coldhead attaches to the copper block and can reach $50 \mathrm{~K}$ in this configuration. Operation at $75 \pm 0.5 \mathrm{~K}$ captures xenon, along with some krypton and argon. Although the cryotrap reduces argon a factor of 100, the residual argon is the major component in the xenon sample and may be the main factor determining the 15 minute transfer time of xenon to the sample bottle. We plan to conduct tests to determine the optimum temperature to retain xenon and reject argon.

The xenon cart SAES GP100 MK5 getters are activated during shot preparation, cooled and left at room temperature to maintain vacuum during xenon transfer to the sample bottle.

The SRS RGA200 monitors several xenon masses, as well as argon. krypton, hydrogen, nitrogen, oxygen, helium, and carbon dioxide. It shows the release of gases during heating of the cryotrap to $135 \mathrm{~K}$ and we use it to determine when to start and end the xenon transfer to the sample bottle based upon the ${ }^{129} \mathrm{Xe}$ signal intensity trends. Transfer of the xenon usually takes 15 minutes and requires periodic replenishment of the liquid nitrogen coolant.

The 50cc sample bottle is custom fabricated (Swagelok), with double valves to prevent air leakage into the sample during transport to the gas handling lab. FIG. 3 is a drawing of the xenon collection cart.

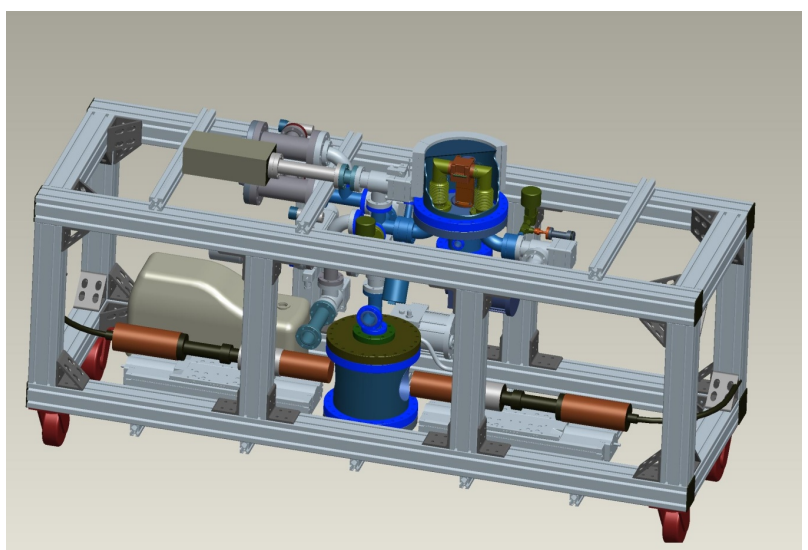

FIG. 3. RAGS xenon collection cart. Noble gas fraction is collected on the copper coldhead, which is then heated and the xenon is released into a sample bottle for subsequent removal and gamma spectroscopy. This drawing shows the addition of a second coldhead for in situ counting of the sample using two germanium detectors. This option will be implemented in Phase II of the project and as of this writing is not yet available.

\section{E. RAGS LaBr Radiation Monitors}

Four LaBr radiation detectors are located on RAGS components. The first two are on the filter cart water trap and the first getter, respectively. The third is on the xenon cart cryotrap 
and the fourth is on the abort tank. These units monitor the radiation levels to inform manned operations after a shot. These detectors have enough resolution at room temperature to identify radioisotopes, however, the diffuse sources in RAGS lead to low count rates. Nonetheless, the detector on the cryotrap was able to identify the xenon radioisotopes produced during the commissioning test (see Section III).

\section{F. Nuclear Counting Facility (NCF)}

We transferred the xenon sample bottle to a gas handling laboratory and prepared it for counting by adding natural isotopic composition xenon to the sample bottle and freezing all the xenon into a smaller calibrated volume attached to a gas gamma cell. The internal volume of the gamma cell had previously been measured by gas expansion from a calibrated volume into the cell. After warming the gas to room temperature, we expanded a portion of the sample into the gamma cell and froze the remainder back into the sample bottle. We calculate the fraction of sample gas in the gas cell from the known volumes and use this factor to determine the number of product atoms in the sample bottle. We started counting the gas cell on an NCF germanium detector within 4 hours of the shot. The counting schedule started with two consecutive 1 hour counts and two consecutive 2 hour counts for the ${ }^{123} \mathrm{Xe}$, followed by two consecutive 8 hour counts. Subsequent 8 hour counts were continued for 2 days for the ${ }^{125} \mathrm{Xe}$. Finally, several 14 day counts provided ${ }^{127} \mathrm{Xe}$ results.

\section{RESULTS}

The collection efficiency of the RAGS system was measured using a manually-operated gas injection system located at port D75 on the equator of the NIF target chamber. One microgram of ${ }^{252} \mathrm{Cf}$, purchased from Oak Ridge National Laboratory, was placed in an inert gas volume to allow the xenon gas produced as fission products $\left({ }^{133,135} \mathrm{Xe}\right)$ to equilibrate with the gas and accumulate. On the day of the injection, the ${ }^{252} \mathrm{Cf}$ volume is attached to a manifold and the inert and fission gases are expanded into two known volumes - a transfer bottle and a gas gamma cell. These volumes are valved off and removed from the manifold. The initial number of xenon fission atoms inside the transfer bottle was determined by gamma counting the gas cell and multiplying by the ratio of volumes. The transfer bottle radioactive xenon was released into the NIF target chamber, and the bottle was returned to measure the residual gas in the same manner as the initial gas. The number of ${ }^{135} \mathrm{Xe}$ atoms introduced into the NIF chamber was $6.7 \times 10^{7}$. RAGS was operated in faux shot mode, as if a shot had occurred but the lasers did not fire. The gas from the NIF chamber was collected through the RAGS apparatus and the xenon was collected in the sample bottle for transfer to the NCF. The gas was prepared as described in section II.F., then gamma counted to measure how much of the initial ${ }^{135} \mathrm{Xe}$ was collected through the entire run from the NIF chamber through RAGS. It was determined that the overall collection efficiency through the entire system was $63.3 \pm 1.1 \%$ of the xenon gas that was initially injected into the chamber. In order to quantify atoms produced on a shot, a tracer injection should be made to measure the sample collection yield.

The first RAGS commissioning test used an exploding pusher target containing enriched ${ }^{124,126,128} \mathrm{Xe}$ as a neutron detector. The goals of the test were to demonstrate collection and decay counting of the radioactive xenon products. During the test, we were able to confirm that the detector gas did mix with the xenon in the air in the target chamber by using the detector cart RGA. Information on the target gas fill for shot N120228001-999 is given in TABLE I. The exploding pusher shell was a $2.1 \mathrm{~mm}$ o.d. glass shell with $0.6 \mathrm{vol} \%$ xenon added to the DT gas fill. The laser energy used for the direct drive shot was $357 \mathrm{~kJ}$ with a $2.85 \mathrm{~ns}$ pulse. The DT neutron yield $(14 \mathrm{MeV})$ was $1.1 \times 10^{13}$ and Tion $=4.6 \mathrm{keV}$.

TABLE I. Xenon gas fill used on shot N120228-001-999.

\begin{tabular}{|c|c|c|}
\hline \hline Isotope & $\begin{array}{c}\text { Abundance } \\
\text { (atom \%) }\end{array}$ & $\begin{array}{c}\text { Target load } \\
\text { (atoms) }\end{array}$ \\
\hline${ }^{124} \mathrm{Xe}$ & 51.5 & \\
${ }^{126} \mathrm{Xe}$ & 44.5 & $3.7 \mathrm{E} 15$ \\
${ }^{128} \mathrm{Xe}$ & 4.0 & $3.2 \mathrm{E} 15$ \\
\end{tabular}

The gases were monitored at both the pre-filter and collection carts using a combination of $\mathrm{LaBr}$ gamma detectors and RGA mass analyzers. The $\mathrm{LaBr}$ detectors were used specifically to track the presence of radioactive xenon through the system. FIG. 4 shows a sample spectrum that was collected during the commissioning shot. The $14 \mathrm{MeV}$ neutron activated ${ }^{123} \mathrm{Xe}$ and ${ }^{125} \mathrm{Xe}$ species are present in the spectrum and the peaks are used to verify the gaseous species of interest have been collected in the gas cylinder before removing it to the nuclear counting facility.

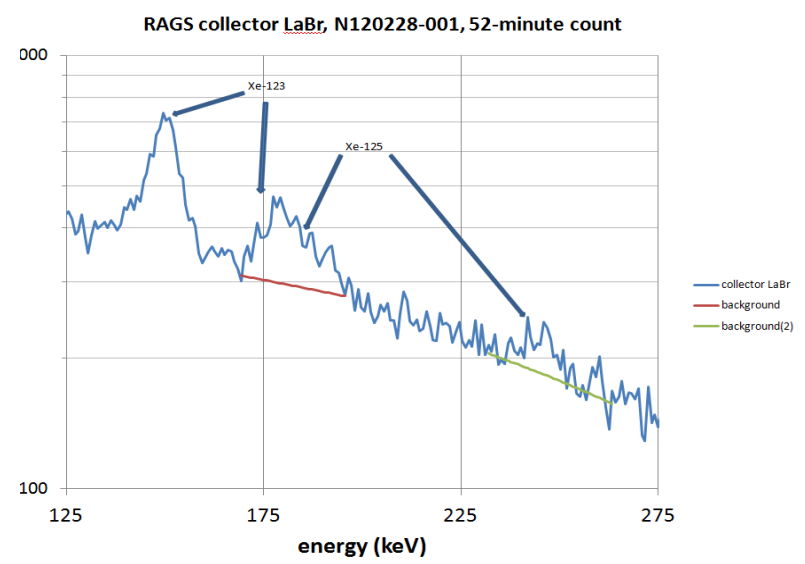

FIG. 4. Sample of gamma-ray spectrum collected during the exploding pusher commissioning shot. The (n,2n) activated products $\left({ }^{123} \mathrm{Xe}\right.$ and $\left.{ }^{125} \mathrm{Xe}\right)$ are visible in the spectrum.

Decay counting results were analyzed with the LLNL GAMANAL $^{3}$ gamma ray analysis program using the xenon nuclear data shown in Table II. Using a xenon recovery value of $63 \%$ measured on the previous injection test, we estimated the numbers of product atoms at Tzero: ${ }^{123} \mathrm{Xe}$ and ${ }^{125} \mathrm{Xe} \sim 2 \times 10^{6}$ atoms each, and ${ }^{127} \mathrm{Xe} \sim 2 \times 10^{5}$ atoms, with a minimum a factor of 2 times lower. The maximum is not known, since it would use the lowest measured recovery value. We plan to make additional 
measurements of collection efficiency using the gas injection system to evaluate the actual range. A sample spectrum from the counting of the final xenon sample in the HPGe detectors is shown in FIG. 5.

TABLE II. Nuclear data used for decay counting. ${ }^{4}$

\begin{tabular}{|c|c|c|c|}
\hline \hline Isotope & $\begin{array}{c}\mathrm{T} 1 / 2 \\
\text { (hours) }\end{array}$ & $\begin{array}{c}\gamma \text { Energy } \\
(\mathrm{keV})\end{array}$ & $\begin{array}{c}\gamma \text { Intensity } \\
(\%)\end{array}$ \\
\hline${ }^{123} \mathrm{Xe}$ & 2.08 & 148.9 & 48.9 \\
& & 178.1 & 14.9 \\
${ }^{125} \mathrm{Xe}$ & \multirow{2}{*}{16.9} & 188.4 & 54.0 \\
& & 243.4 & 30.1 \\
${ }^{127} \mathrm{Xe}$ & \multirow{2}{*}{873.6} & 172.1 & 25.5 \\
& & 220.9 & 68.3 \\
& & 375 & 17.2 \\
\hline
\end{tabular}

If we had measured a detector gas recovery value specific for this shot, we could have calculated $(n, 2 n)$ cross section values. We were unable to measure recovery due to an overpressure shutdown of the xenon cart RGA. However, we calculated normalized production ratios for the $(n, 2 n)$ reaction products by scaling counting atom ratios with the target isotope fractions and assuming small $(n, \gamma)$ contributions vs. (n,2n). We compare these ratios to previously reported $(n, 2 n)$ cross section ratios and find that our ratio values are lower, but consistent with the two ratio values being the same (TABLE III).

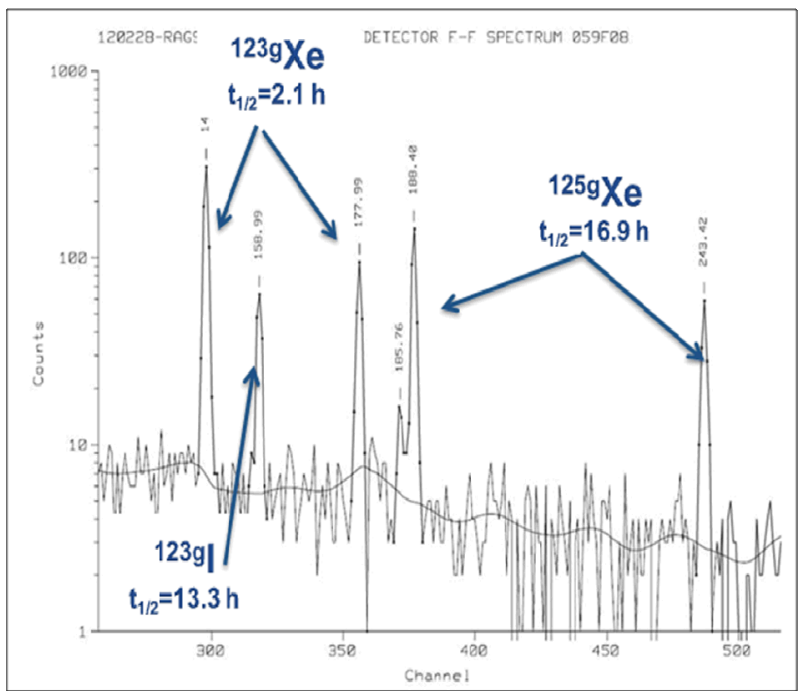

FIG. 5. Sample gamma spectrum taken with a high purity germanium detector. This spectrum was taken of the xenon gas sample that was collected after shot N120228-001-999 after removing it from RAGS.
TABLE III. Normalized n,2n Production ratios with $14 \mathrm{MeV}$ DT neutrons. It was assumed that the $n, \gamma$ contribution to the production of these isotopes was negligible.

\begin{tabular}{|c|c|c|}
\hline \hline Isotope & Normalized Ratio & Cross Section Ratio ${ }^{5}$ \\
\hline${ }^{125} \mathrm{Xel}{ }^{123} \mathrm{Xe}$ & $1.22 \pm 0.05$ & $1.48 \pm 0.21$ \\
${ }^{127} \mathrm{Xe}{ }^{123} \mathrm{Xe}$ & $1.24 \pm 0.17$ & $1.45 \pm 0.21$ \\
\hline
\end{tabular}

\section{CONCLUSION}

The RAGS system has been shown to effectively collect a large fraction of the gas load from the NIF chamber following a shot. The final noble gas samples produced after purification and isolation are of diagnostic quality that could be used to measure $\rho r$ and mix features in NIF targets. Additional measurements of the collection efficiency are required. The addition of germanium detectors to the xenon collection cart would allow for in-situ measurement of short-lived radioisotopes. Mix diagnostics require either simultaneous injection of a noble gas tracer into the chamber or co-loading of a tracer into the capsule itself.

\section{ACKNOWLEDGEMENTS}

This work was performed under the auspices of the U.S. Department of Energy by Lawrence Livermore National Laboratory under Contract DE-AC52-07NA27344.

\section{REFERENCES}

${ }^{1}$ D. A. Shaughnessy, C. Cerjan, K. J. Moody, L. Bernstein, R. Hoffman, M. A. Stoyer, R. Fortner, and D. Schneider, Lawrence Livermore National Laboratory Report LLNL-TR-472959 (2011).

${ }^{2}$ Names of commercial products and vendors are used for informational purposes only to describe equipment used on the system and does not constitute endorsement by the authors nor by Lawrence Livermore National Laboratory.

${ }^{3}$ R. Gunnink and J. B. Niday, "Computerized Quantitative Analysis by Gamma-Ray Spectrometry: Vol 1. Description of the GAMANAL Program," Lawrence Livermore National Laboratory Report UCRL-51061, Vol. I, March 1, 1972.

${ }^{4}$ R. B. Firestone, Table of Isotopes, $8^{\text {th }}$ ed. (Wiley-Interscience, 1999).

${ }^{5}$ R. A. Sigg and P. K. Kuroda, Nuclear Science and Engineering, 60, 235-238, 1976, values at $14.6 \mathrm{MeV}$. 